\title{
Trk inhibitor GNF-5837 suppresses the tumor growth, survival and migration of renal cell carcinoma
}

\author{
YICHENG CHEN $^{1}$, HUAN WANG ${ }^{1}$, YUANLEI CHEN ${ }^{1}$, \\ MINGCHAO WANG ${ }^{1}$, GUOQING DING ${ }^{1}$ and TAO LI ${ }^{2}$ \\ ${ }^{1}$ Department of Urology, Sir Run-Run Shaw Hospital, College of Medicine, \\ Zhejiang University, Hangzhou, Zhejiang 310016; ${ }^{2}$ School of Medicine, \\ Hunan Normal University, Changsha, Hunan 410081, P.R. China
}

Received December 13, 2018; Accepted July 3, 2019

DOI: $10.3892 / o r .2019 .7296$

\begin{abstract}
The clinical treatment of renal cell carcinoma (RCC) remains a major challenge. A number of novel agents and therapeutic strategies are currently undergoing active investigation. In the present study, we investigated the potential of GNF-5837, a Trk inhibitor, in the treatment of RCC. Tropomyosin-related kinases (Trk), a family of neurotrophin receptors, are vital for neural development and have also been identified as prognostic markers in malignancies of diverse origins. In the present study, we demonstrated that GNF-5837, an inhibitor of TrkA and TrkB, suppressed the cell viability of renal carcinoma 7860 and Caki-2 cells in a concentration-dependent manner. GNF-5837 treatment led to decreased activities of TrkA and TrkB signaling, accompanied by reduced phosphorylation levels of AKT and extracellular signal-regulated kinase (ERK) kinases, which was detected by western blot assay. GNF-5837 induced G0/G1-phase arrest and apoptosis. Consistently, GNF-5837 affected the expression of p21, c-Myc, and survivin proteins. Meanwhile, a wound healing assay showed that GNF-5837 inhibited the migration ability of RCC cells by impairing Rac1 activity. GNF-5837 also enhanced the cytotoxic effects of sunitinib via inhibition of ERK kinase. Taken together, these results identify the pharmacological potential of targeting Trk signaling as a therapeutic strategy for RCC.
\end{abstract}

Correspondence to: Professor Guoqing Ding, Department of Urology, Sir Run-Run Shaw Hospital, College of Medicine, Zhejiang University, 3 East Qingchun Road, Hangzhou, Zhejiang 310016, P.R. China

E-mail: 3196014@zju.edu.cn

Dr Tao Li, School of Medicine, Hunan Normal University, 371 Tongzipo Road, Changsha, Hunan 410081, P.R. China

E-mail: litao7997@hunnu.edu.cn

Key words: GNF-5837, renal cell carcinoma, cytotoxicity, tropomyosin-related kinase

\section{Introduction}

Renal cell carcinoma (RCC) is the most common kidney cancer subtype. More than 100,000 cases are diagnosed worldwide per year, and RCC accounts for approximately $2-5 \%$ of all adult neoplastic diseases and its incidence is rising (1). RCC originates from the tubular structures of the kidney and comprises a heterogeneous group of malignancies. RCC can be classified into four major histological cell types, of which clear cell RCC (ccRCC) is the most common (75-80\%) (2). Other types include papillary (10-15\%), chromophobe (5\%) and collecting duct (1\%) RCC. Metastasis may be present at the time of RCC diagnosis and such cases are resistant to radiotherapy, chemotherapy and targeted therapy. Despite great efforts in the field of RCC therapy, RCC remains an incurable disease and the median survival period of patients with advanced RCC is approximately 26 months (3). Therefore, there is a compelling demand to identify both molecular targets and novel drugs with which to treat this disease.

Inhibition of vascular endothelial growth factor (VEGF) receptors is a promising approach for the targeted therapy of RCC. Antiangiogenic drugs for RCC treatment such as sunitinib, pazopanib, sorafenib and axitinib have recently received particular attention (3-5). Sunitinib is a small-molecule inhibitor of multiple receptor tyrosine kinases (RTKs), that inhibits the family of VEGF receptors (VEGFR-1, VEGFR-2 and VEGFR-3), PDGF receptors (PDGFR- $\alpha$ and PDGFR- $\beta$ ), FLT3, the stem cell growth factor receptor KIT and RET. The repressive effect of sunitinib on tumor growth and invasion is mainly achieved by antagonizing the activation of VEGFR and PDGFR, two crucial players in the pathogenesis of RCC. Sunitinib treatment for metastasized RCC contributes to a significantly longer progression-free survival and overall survival. Although sunitinib has become the mainstay of RCC treatment, the occurrence of resistance is a main obstacle overshadowing its clinical benefit. Additional underlying therapeutic targets for RCC include mammalian target of rapamycin (mTOR) kinase, immune checkpoint proteins, indoleamine 2,3-dioxygenase and transforming growth factor- $\beta$ (3-5).

As tumor cells may escape the inhibition of VEGF signaling using other parallel pathways as growth and survival signals (6), it is necessary to seek accessory pathways to develop new treatment approaches and avoid chemotherapeutic 
resistance. Tropomyosin-related kinases (Trks) are a family of receptor tyrosine kinases consisting of 3 isoforms (TrkA, TrkB and $\operatorname{TrkC}$ ) that are activated by neurotrophins. In the present study, we demonstrated that GNF-5837, an inhibitor of TrkA and TrkB, reduced the cell viability of renal carcinoma cells via inducing cell cycle arrest and apoptosis. Meanwhile, GNF-5837 also suppressed the migration ability of renal carcinoma cells. Taken together, these results identify the potential pharmacological merit of a Trk inhibitor for the chemotherapy of RCC.

\section{Materials and methods}

Reagents. GNF-5837 and sunitinib were purchased from Selleck Chemicals (Houston, TX, USA).

Cell culture. The human RCC cell lines 7860 and Caki-2 were purchased from the Shanghai Institute of Cell Biology (Shanghai, China) and were cultured in Roswell Park Memorial Institute (RPMI)-1640 medium supplemented with $10 \%$ fetal bovine serum (FBS; Gibco/Thermo Fisher Scientific, Inc.), penicillin $(100 \mathrm{U} / \mathrm{ml})$, and streptomycin $(100 \mu \mathrm{g} / \mathrm{ml})$ under a humidified atmosphere containing $5 \% \mathrm{CO}_{2}$ maintained at $37^{\circ} \mathrm{C}$.

MTS assay. Cell viability was determined using the CellTiter $96{ }^{\circledR} \mathrm{AQ}_{\text {ueous }}$ One Solution Cell Proliferation assay kit (Promega, Madison, WI, USA). Cells $\left(5 \times 10^{3}\right)$ were seeded into 96-well plates and treated with GNF-5837 or other agents for the depicted time intervals. After treatment, $10 \mu \mathrm{l}$ MTS (Promega) was added into each well for a 2-h incubation. The absorbance was measured using a model ELX800 Micro Plate Reader (Bio-Tek Instruments, Winooski, VT, USA) at $490 \mathrm{~nm}$.

EdU incorporation assay. Cell proliferation was determined by incorporation of 5-ethynyl-2'-deoxyuridine (EdU) using an EdU Cell Proliferation Assay kit (Guangzhou RiboBio Co., Ltd., Guangzhou, China) as previously described (7). Cells were cultured in triplicate in 24-well plates at a density of $5 \times 10^{4}$ and treated with GNF-5837 for $48 \mathrm{~h}$ at $37^{\circ} \mathrm{C}$, and then $50 \mathrm{mM}$ EdU was added and incubated for an additional $2 \mathrm{~h}$ at $37^{\circ} \mathrm{C}$. The cells were fixed with $4 \%$ formaldehyde for $30 \mathrm{~min}$ at room temperature and treated with $2 \mathrm{mg} / \mathrm{ml}$ glycine for $5 \mathrm{~min}$. After washing with phosphate-buffered saline (PBS), the cells were treated with $0.5 \%$ Triton $\mathrm{X}-100$ for $10 \mathrm{~min}$ at room temperature for permeabilization. Apollo ${ }^{\circledR}$ reaction cocktail $(100 \mu \mathrm{l})$ was added to each well and incubation was carried out for $30 \mathrm{~min}$ in the dark at room temperature. After washing with Triton X-100, the cells were stained with $100 \mu$ l Hoechst 33342 for 30 min and visualized under a fluorescence microscope (Olympus Corp., Japan) at x200 magnification. A total of 100 cells from the different groups was analyzed for EdU labeling in this study.

Flow cytometric analysis. To assess cell cycle progression by flow cytometry, 7860 cells $\left(2 \times 10^{5}\right)$ after GNF-5837 treatment were suspended in $100 \mu \mathrm{l}$ of PBS, and $200 \mu \mathrm{l}$ of $95 \%$ ethanol was added. The cells were then incubated at $4^{\circ} \mathrm{C}$ for $1 \mathrm{~h}$, washed with PBS, resuspended in $250 \mu \mathrm{l}$ of $1.12 \%$ sodium citrate buffer (pH 8.4) together with $12.5 \mu \mathrm{g}$ of RNase, and incubated at $37^{\circ} \mathrm{C}$ for an additional $30 \mathrm{~min}$. The cellular DNA was then stained by $250 \mu \mathrm{l}$ propidium iodide (PI) for $30 \mathrm{~min}$ at room temperature. Red fluorescence emitted from the PI-DNA complex was analyzed at $488 \mathrm{~nm} / 600 \mathrm{~nm}$ (excitation/emission wavelength) using a FACScan flow cytometer (BD LSR II). The data of relative DNA content were analyzed by ModFit LT software package (https://www.vsh.com/products/mflt/mfFeatures.asp) to reveal cell cycle distribution.

Apoptosis assay. 7860 and Caki- 2 cells $\left(2 \times 10^{5}\right)$ were seeded in 6-well plates and allowed to attach overnight. After GNF-5837 treatment for $48 \mathrm{~h}$, cells were harvested and resuspended in $200 \mu \mathrm{l}$ binding buffer after washing twice with cold PBS. Then, apoptotic cells were assessed by an Annexin-V apoptosis detection kit (MultiSciences Biotech, Hangzhou, China) according to the manufacturer's protocol. The cell suspension was incubated with $10 \mu$ l Annexin V-fluorescein isothiocyanate (FITC) stock solution for $30 \mathrm{~min}$ at $4^{\circ} \mathrm{C}$ in the dark, and then incubated with $5 \mu \mathrm{l}$ PI solution for $5 \mathrm{~min}$. Cell samples were analyzed by flow cytometry (BD LSR II; BD Biosciences, Franklin Lakes, NJ, USA) and apoptotic cell fractions were determined.

Real-time polymerase chain reaction ( $q P C R)$. Total RNA was isolated using the RNAiso Plus (Takara Biotechnology, Dalian, China), and cDNA was prepared using Transcriptor First Strand cDNA Synthesis kit (Roche) according to the manufacturer's instructions. Quantitative reverse transcriptase PCR (RT-qPCR) was performed to quantify the expression of the genes of interest using LightCycler ${ }^{\circledR} 480$ SYBR-Green I Master (Roche, Indianapolis, IN, USA) according to the manufacturer's protocol on Roche light cycler version 3.5. The following thermal profile was applied: 1 cycle at $95^{\circ} \mathrm{C}$ for $10 \mathrm{~min}, 40$ cycles at $95^{\circ} \mathrm{C}$ for $10 \mathrm{sec}, 60^{\circ} \mathrm{C}$ for $30 \mathrm{sec}$, and $72^{\circ} \mathrm{C}$ for $15 \mathrm{sec}$. The level of expression of each target gene was calculated using the $2^{-\Delta \Delta \mathrm{Cq}}$ method (8). The relative amount of each mRNA was normalized to GAPDH. Each sample was examined in triplicate. qPCR analysis was performed with the following primers: Survivin 5'-GAGGCTGGCTTCATCCACTG-3' (forward) and 5'-GCA CTTTCTTCGCAGTTTCCTC-3' (reverse); Bcl2 5'-TCGCCC TGTGGATGACTGA-3' (forward) and 5'-CAGAGACAGCCA GGAGAAATC-3' (reverse); Bax 5'-TGGCAGCTGACATGT TTTCTGAC-3' (forward) and 5'-TCACCCAACCACCCTGGT CTT-3' (reverse); GAPDH 5'-CTCACCGGATGCACCAAT GTT-3' (forward) and 5'-CGCGTTGCTCACAATGTTCAT-3' (reverse). The designed primers were synthesized by Sangon Biotech (Shanghai, China).

Western blot analysis. The cells $\left(1 \times 10^{6}\right)$ were washed with cold PBS and lysed on ice in $100 \mu 1$ modified radioimmunoprecipitation assay (RIPA) buffer (50 mM Tris- $\mathrm{HCl}, \mathrm{pH} 7.4$, $1 \%$ NP-40, 0.25\% Na-deoxycholate, $150 \mathrm{mM} \mathrm{NaCl}, 1 \mathrm{mM}$ $\mathrm{Na}_{3} \mathrm{VO}_{4}$, and $1 \mathrm{mM} \mathrm{NaF}$ ) containing protease inhibitors [100 $\mu \mathrm{M}$ phenylmethylsulfonyl fluoride, $10 \mu \mathrm{M}$ leupeptin, $10 \mu \mathrm{M}$ pepstatin and $2 \mathrm{mM}$ ethylenediaminetetraacetic acid (EDTA)]. The protein content was measured using a BCA Protein Assay kit (Beyotime, Shanghai, China). The proteins were separated by $10 \%$ SDS-PAGE (sodium dodecyl sulphate-polyacrylamide gel electrophoresis) and transferred to polyvinylidene fluoride (PVDF) membranes (Millipore Corp., Bedford, MA, USA), and then blotted with the specific antibodies. Antibodies against phosphorylated TrkA (cat. no. AP0492; dilution 1:500), total TrkA (cat. no. A15618; dilution 1:500), phosphorylated TrkB (cat. no. AP0423; 
A

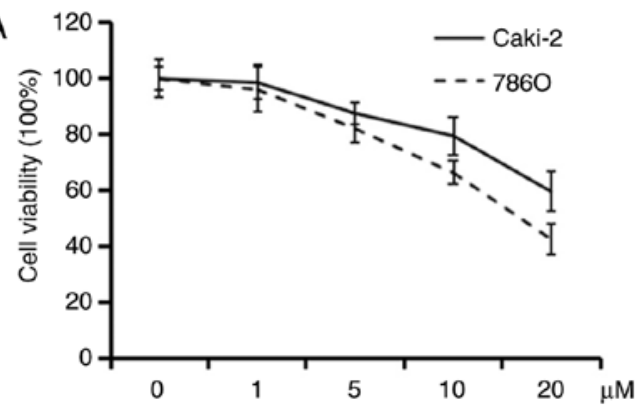

B

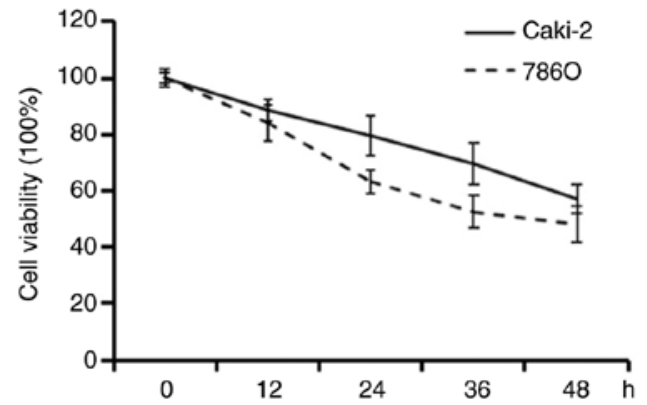

Figure 1. GNF-5837 reduces cell viability in RCC cells. (A) Cell viability was estimated by MTS assay after Caki-2 and $786 \mathrm{O}$ cells were incubated with increasing concentrations of GNF-5837 (0-20 $\mu \mathrm{M})$ for $48 \mathrm{~h}$. DMSO was used as a control. (B) The time-response curve of $20 \mu \mathrm{M}$ GNF-5837 on cell viability of Caki-2 and 7860 cells. Data in A and B represent the mean \pm SD of three independent experiments. RCC, renal cell carcinoma.

dilution 1:500), total TrkB (cat. no. A2099; dilution 1:500), and $\beta$-actin (cat. no. AC026; dilution 1:500) were obtained from ABclonal Biotechnology (Wuhan, China). Antibodies against phosphorylated Rac1 (cat. no. 2461; dilution 1:1,000), total Rac1 (cat. no. 4651; dilution 1:1,000), phosphorylated ERK (cat. no. 9101; dilution 1:1,000), total ERK (cat. no. 4695; dilution 1:1,000), phosphorylated AKT (cat. no. 4060; dilution 1:1,000), and total AKT (cat. no. 9272; dilution 1:1,000) were obtained from Cell Signaling Technology (Danvers, MA, USA). Antibodies specific for p21 (cat. no. sc-6246; dilution 1:200), c-Myc (cat. no. sc-40; dilution 1:200), poly(ADP-ribose) polymerase-1 (PARP-1) (cat. no. sc-56197; dilution 1:200), and survivin (cat. no. sc-17779; dilution 1:200) were purchased from Santa Cruz Biotechnology (Santa Cruz, CA, USA). The secondary antibodies were horseradish peroxidase conjugated goat anti-rabbit IgG (cat. no. AS014; dilution 1:2,000; ABclonal Biotechnology) and anti-mouse IgG (cat. no. AS003; dilution 1:2,000; ABclonal Biotechnology) antibodies. Detection of specific proteins was carried out with an ECL chemiluminescence detection kit (Vigorous, Beijing, China) according to the manufacturer's instructions.

Wound healing assay. $786 \mathrm{O}$ and Caki-2 cells were seeded in 6 -well plates for the wound healing assay. When monolayer cells grew to about $80 \%$ confluence, they were wounded by the tip of a sterile $200-\mu 1$ micropipette. Cells were washed with PBS (pH 7.4) for 3 times and then cultured with complete medium (10\% FBS) containing $20 \mu \mathrm{M}$ GNF-5837. The migration of the cells at the edge of the scratch was analyzed at $24 \mathrm{~h}$. Images were captured by an inverted microscope (Olympus Corporation, Japan) at x10.

Statistical analysis. All data in this study are displayed as means $\pm \mathrm{SD}$ ( $\mathrm{n} \geq 3$ repeats). Comparisons were analyzed by Student's t-test or one-way analysis of variance (ANOVA) followed by the Student-Newman-Keuls (SNK) test. The significance was analyzed with SPSS 10.0 software (SPSS, Inc., Chicago, IL, USA) and a P-value $<0.05$ was considered to indicate a statistically significant result.

\section{Results}

GNF-5837 decreases the cell viability of the RCC cell lines. In order to evaluate the effects of a Trk inhibitor GNF-5837 on RCC cell lines, cell viability was tested in Caki-2 and
A
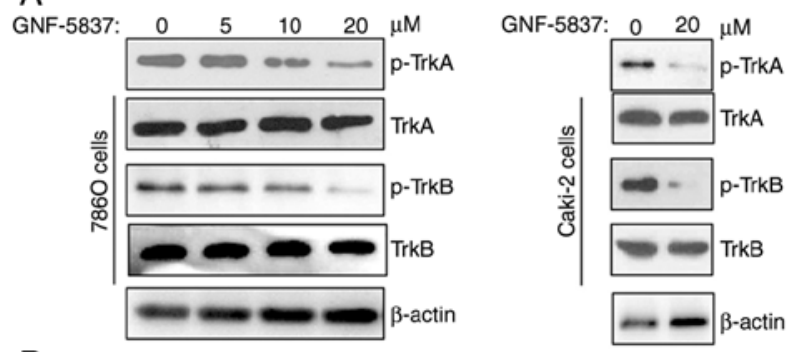

B
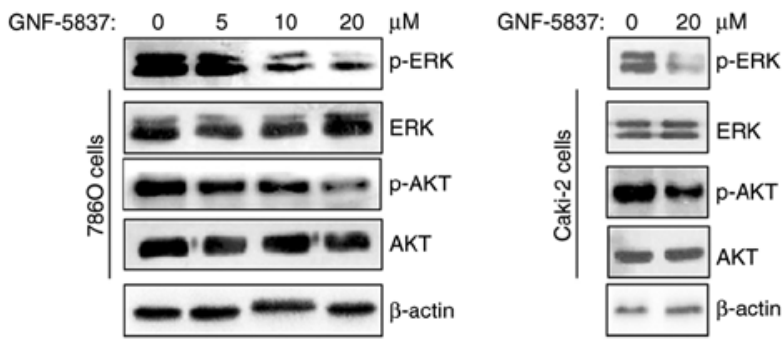

Figure 2. GNF-5837 inhibits Trks and downstream kinases. (A) and (B) 7860 and Caki-2 cells were treated with GNF-5837 at the indicated concentrations for $48 \mathrm{~h}$, and then cells were harvested and western blot analysis was performed for the evaluation of the phosphorylation levels (p-) of TrkA and TrkB (A) and ERK and AKT (B). Representative images from at least three independent experiments are shown. Trks, tropomyosin-related kinases.

7860 cell lines by MTS assay. The 7860 cell line has many characteristics of ccRCC. Although the Caki-2 cell line was primarily recognized as a ccRCC cell line, an accumulating amount of data showed that it is a cell line of papillary RCC. After exposure to elevated concentrations of GNF-5837 $(0-20 \mu \mathrm{M})$ for $48 \mathrm{~h}$, both of the tested RCC cell lines showed a dose-dependent decrease in cell viability (Fig. 1A). Our results revealed that GNF-5837 at $20 \mu \mathrm{M}$ exerted a significant effect on cell viability. Compared with the DMSO-treated control, the viability of the Caki-2 and 7860 cells was decreased to about 57.1 and $48.2 \%$ after treatment with $20 \mu \mathrm{M}$ GNF-5837 for $48 \mathrm{~h}$, respectively. MTS assays also showed that exposure to $20 \mu \mathrm{M}$ GNF-5837 resulted in marked time-dependent reduction in cell viability (Fig. 1B).

GNF-5837 inhibits Trks and its downstream signaling pathways. Subsequently, we evaluated the inhibition of GNF-5837 on Trk signaling in $786 \mathrm{O}$ cells. As shown in Fig. 2A, GNF-5837 treatment $(0-20 \mu \mathrm{M})$ for $48 \mathrm{~h}$ in 7860 cells effectively 
A
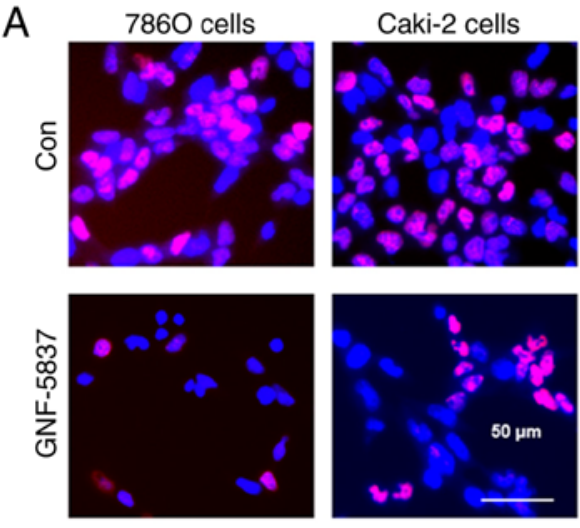

D
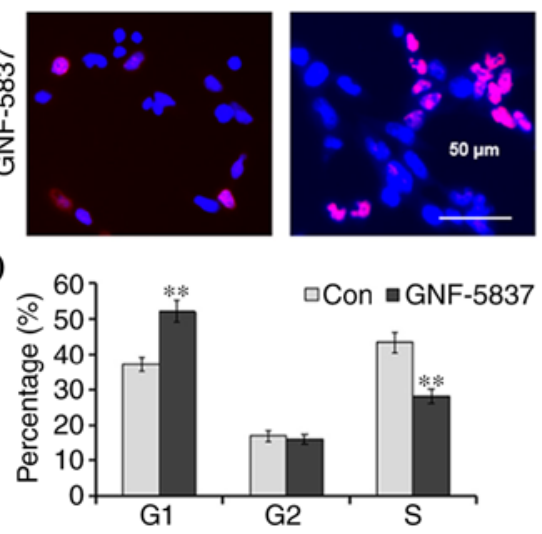

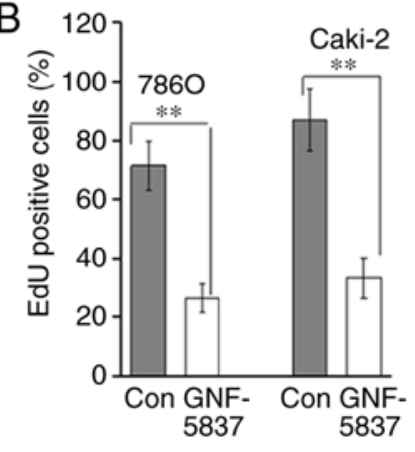

E

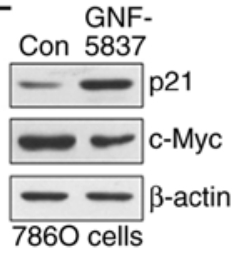

$\mathrm{C}$

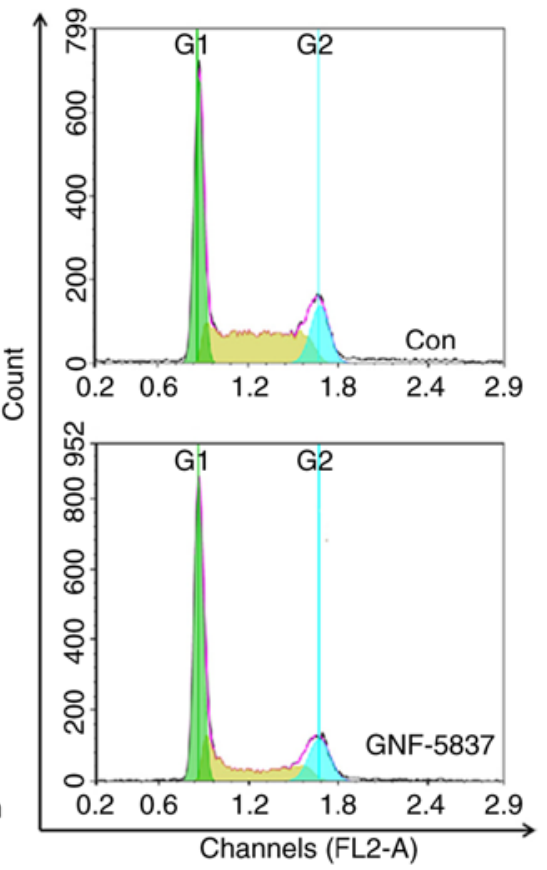

Figure 3. GNF-5837 suppresses the cell growth of RCC cells. (A) EdU incorporation assay was analyzed by fluorescence microscopy in $786 \mathrm{O}$ and Caki-2 cells treated with $20 \mu \mathrm{M}$ GNF-5837 for $48 \mathrm{~h}$. Nuclei were visualized with Hoechst 33342. Magnification, x200. (B) The EdU incorporation rate was expressed as the ratio of EdU-positive cells to total Hoechst 33342-positive cells. A total of 100 cells from multiple visual fields of one group was analyzed for EdU labeling in this study. Data represent mean \pm SD of three independent experiments. ${ }^{* *} \mathrm{P}<0.01$ vs. the control (Con). (C) After GNF-5837 treatment for $48 \mathrm{~h}, 786 \mathrm{O}$ cells were stained with propidium iodide (PI) and subjected to cell cycle analysis by flow cytometry. One representative experiment out of three is shown. (D) Statistical analysis of the cell percentages for the cell cycle distribution. Data represent mean $\pm \mathrm{SD}$ of three independent experiments. ${ }^{* *} \mathrm{P}<0.01$ vs. the control (Con). (E) Changes in cell-cycle regulatory proteins after GNF-5837 treatment for $48 \mathrm{~h}$. Representative images from at least three independent experiments are shown. RCC, renal cell carcinoma.

decreased the phosphorylation of TrkA and TrkB signaling in a dose-dependent manner. High concentration of GNF-5837 $(20 \mu \mathrm{M})$ produced similar effects in Caki-2 cells. Activation of Trk signaling has been demonstrated to affect cell growth, survival, and differentiation via modulating the PI3K/AKT and MAPK/ERK signaling pathways. Thus, we further assessed the effect of GNF-5837 treatment on Trk downstream molecules including AKT and ERK kinases. As shown in Fig. 2B, GNF-5837 directly interrupted the phosphorylation activation of AKT and ERK kinases in a dose-dependent manner in 7860 cells. Similar results were also observed in Caki-2 cells. Overall, these findings indicate that GNF-5837 treatment inhibits Trk kinases and downstream signaling pathways.

GNF-5837 suppressed the cell growth of RCC cells. Next, we investigated the effect of GNF-5837 treatment on cell growth. Firstly, EdU incorporation assay was used to detect DNA synthesis. After incubation with $20 \mu \mathrm{M} \mathrm{GNF}-5837$ for $48 \mathrm{~h}$, the number of EdU-positive 7860 and Caki-2 cells was significantly decreased (Fig. 3A). For example, EdU-incorporating cells accounted for as much as $71.4 \%$ of the total 7860 cells in the control group, while only $26.3 \%$ cells were positive for EdU staining after GNF-5837 treatment for 48 h (Fig. 3B). These results indicated that the Trk inhibitor inhibited the proliferation of RCC cells. Secondly, the cell cycle profile was analyzed using propidium iodide staining and flow cytometry after treatment with $20 \mu \mathrm{M}$ GNF-5837 for $48 \mathrm{~h}$ in 7860 cells. Compared with the control, GNF-5837 treatment significantly decreased the cell populations in the S phase (Fig. 3C and D). Meanwhile, GNF-5837 treatment mainly induced cell cycle arrest in the G0/G1 phase. Coincidently, GNF-5837 treatment upregulated the expression of p21 protein, a CDK inhibitor, in both 7860 and Caki-2 cell lines (Fig. 3E). In comparison, GNF-5837 exerted a moderate inhibition on the expression of c-Myc, a master regulator of cell division.

GNF-5837 induces cell apoptosis. Since GNF-5837 was found to inhibit the viability and growth of Caki-2 and 7860 cells, we investigated whether GNF-5837 induces the apoptosis of RCC cells. After incubation with $20 \mu \mathrm{M}$ GNF-5837 for $48 \mathrm{~h}$, 7860 and Caki- 2 cells were stained with Annexin V-FITC and propidium iodide (PI) and analyzed by flow cytometry. As shown in Fig. 4A, GNF-5837 increased the apoptotic populations (Annexin V-FITC-positive cells) to 20.6 \pm 2.1 and $15.5 \pm 2.9 \%$ in the 7860 and Caki-2 cells $(\mathrm{P}<0.01$, vs. control), respectively. Next, immunoblotting assay was performed to investigate the expression of cleaved PARP-1, a hallmark of apoptosis. As expected, incubation with $20 \mu \mathrm{M}$ GNF-5837 triggered the cleavage of PARP-1 in 7860 and Caki- 2 cells (Fig. 4B). Furthermore, apoptosis-related genes were examined by qPCR assay. As shown in Fig. 4C, the expression of anti-apoptotic survivin gene (BIRC5) in 7860 cells was significantly downregulated by GNF-5837 treatment. Bcl2 expression was slightly altered. Conversely, the pro-apoptotic protein Bax was significantly induced. Immunoblotting assay further confirmed that GNF-5837 treatment suppressed the abundance of the survivin protein (Fig. 4D). 
A

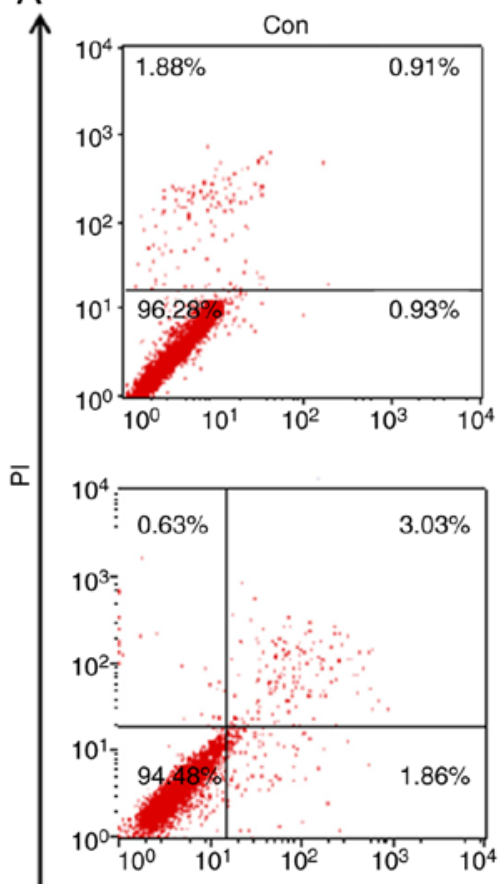

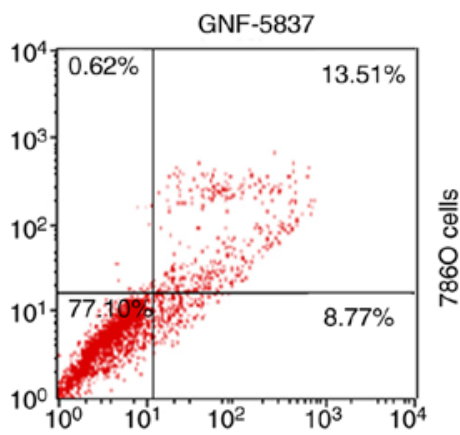
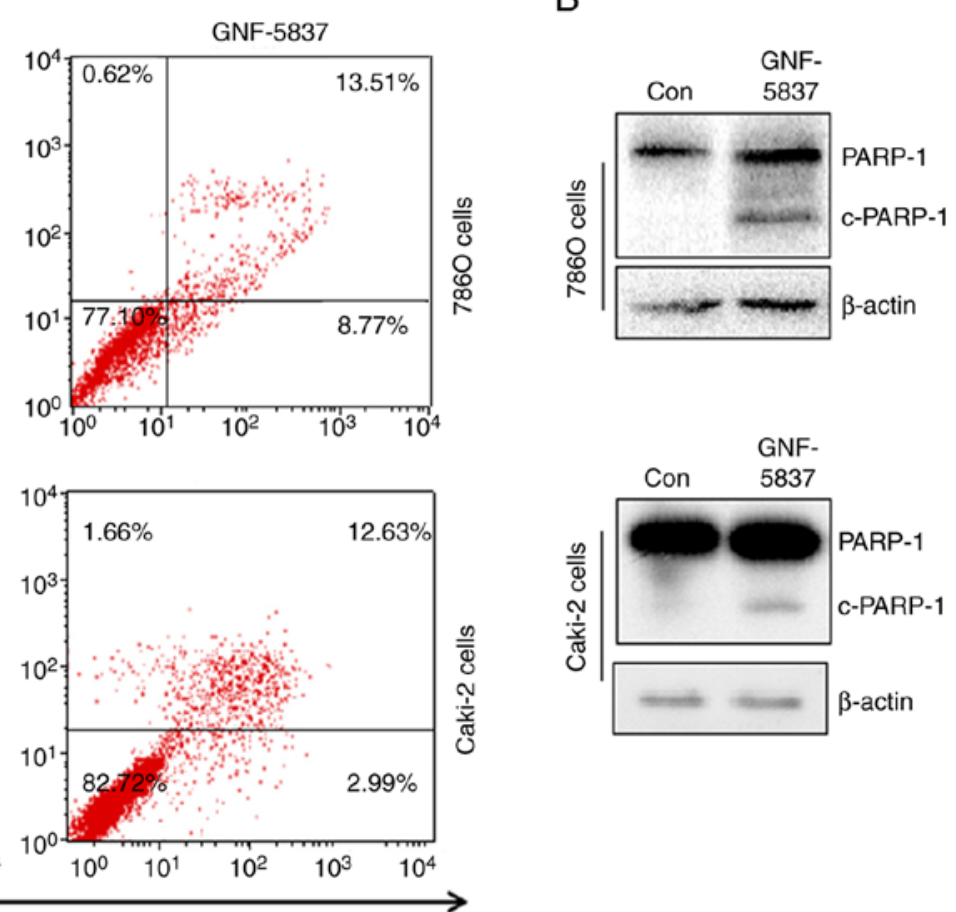

Annexin V-FITC

C

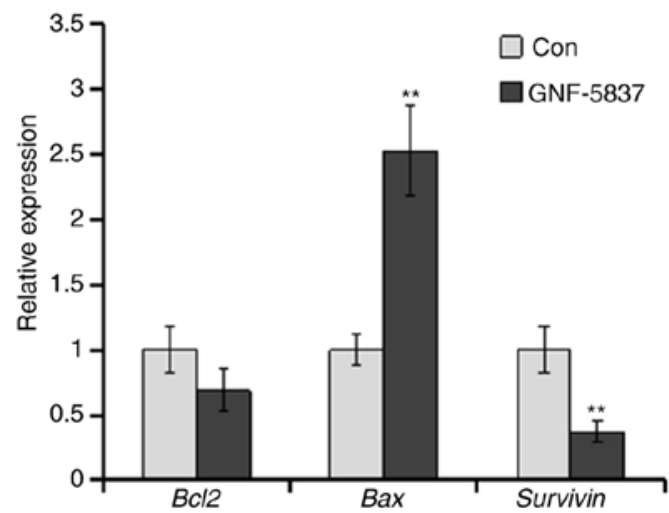

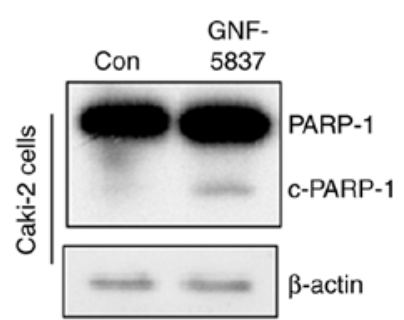

Figure 4. GNF-5837 affects cell apoptosis. (A) Flow cytometric analysis of apoptosis was determined using Annexin V-FITC/PI staining in 786O and Caki-2 cells treated with $20 \mu \mathrm{M}$ GNF-5837 for $48 \mathrm{~h}$. Data are typical of three similar experiments. The percentage of Annexin V-FITC and/or PI positive cells was depicted with cytofluorometer quadrant graphs. (B) GNF-5837 treatment induced the cleavage of PARP-1 as indicative of apoptosis. (C) qPCR was performed to estimate expression alterations of anti-apoptotic and pro-apoptotic genes after GNF-5837 treatment for $48 \mathrm{~h}$ in $786 \mathrm{O}$ cells. ${ }^{* * *} \mathrm{P}<0.01 \mathrm{vs}$. the control (Con). (D) Western blot analysis was performed to verify the alteration of survivin protein. Representative images from at least three independent experiments are shown.

GNF-5837 potentiates sunitinib-induced cytotoxicity. Subsequently, we estimated the combined effects of GNF-5837 together with sunitinib, a tyrosine kinase inhibitor in the treatment of metastatic RCC. By comparison, sunitinib is a strong apoptosis inducer and alone can trigger a majority of cells to undergo apoptosis. Thus, we shortened the treatment period to reduce the toxicity of sunitinib, thereby to manifest the cooperative effect of GNF-5837. $786 \mathrm{O}$ cells were exposed to $20 \mu \mathrm{M}$ sunitinib together with increasing concentrations of GNF-5837 for $24 \mathrm{~h}$. Following treatment, MTS assay was performed. As shown in Fig. 5A, sunitinib alone decreased the cell viability of 7860 cells by $42.9 \%$. When the cells were cotreated with 10 or $20 \mu \mathrm{M}$ GNF-5837, the inhibition rate increased to 60.7 and $68.8 \%$ following cotreatment, respectively. Similar results were obtained for the Caki-2 cells. The results from flow cytometry also demonstrated that cotreatment with GNF-5837 and sunitinib greatly triggered cell apoptosis in the 7860 cells (Fig. 5B). Apoptosis occurred in $13.9 \pm 1.2,31 \pm 5.3$ and $60 \pm 8.8 \%$ of $786 \mathrm{O}$ cells in the GNF-5837, sunitinib and combined treatment groups, respectively. PARP-1 cleavage in 7860 and Caki- 2 cells was increased after the combined treatment (Fig. 5C). These results indicate that there is an enhanced antitumor effect of sunitinib and GNF-5837.

GNF-5837 cooperates with sunitinib to inhibit the ERK cascade. To further ascertain the underlying mechanism involving the the synergy of GNF-5837 and sunitinib, we examined the alteration of Trk, ERK and AKT signals in 7860 cells under different circumstances. As shown in Fig. 6A, sunitinib 
A
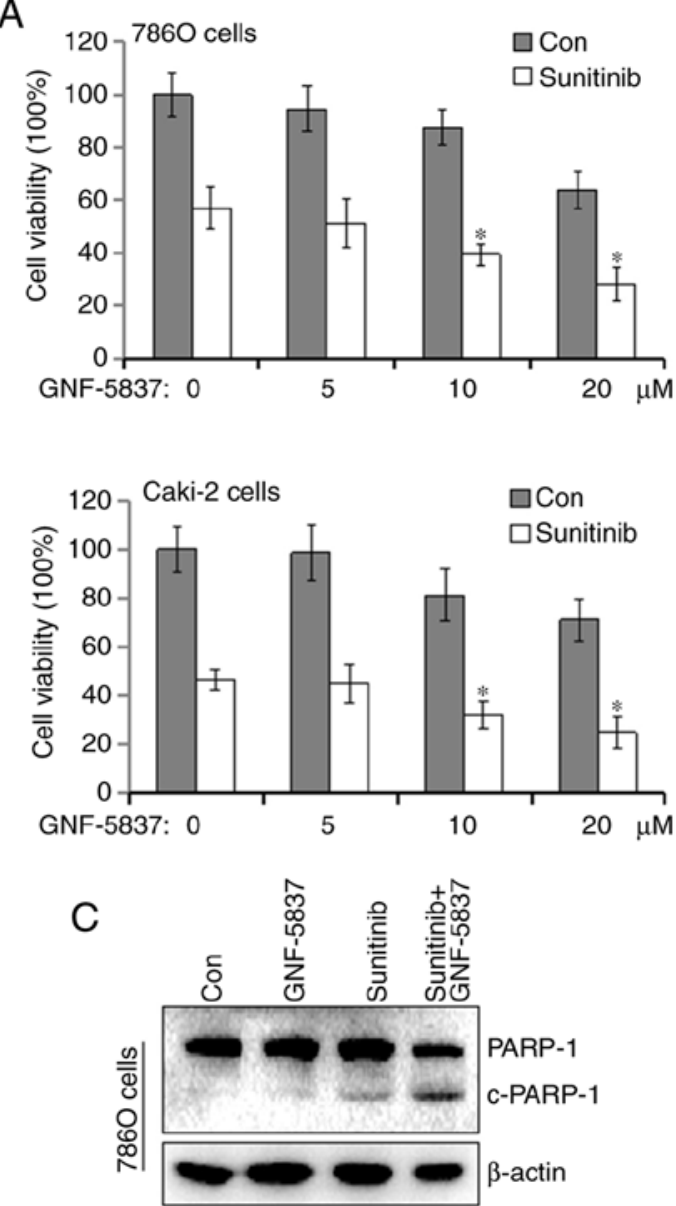

$\mathrm{B}$
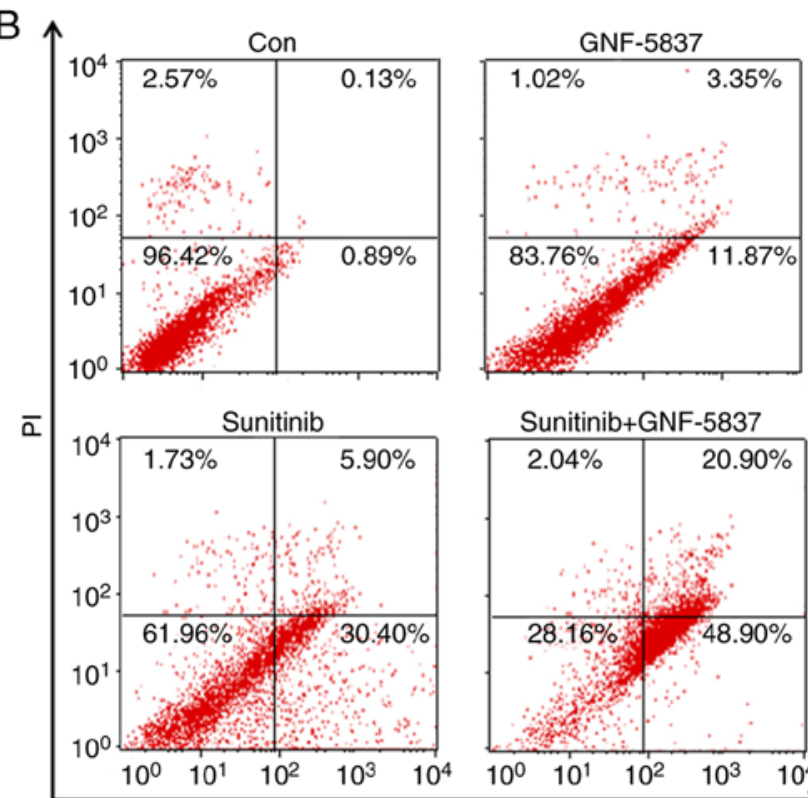

Sunitinib+GNF-5837

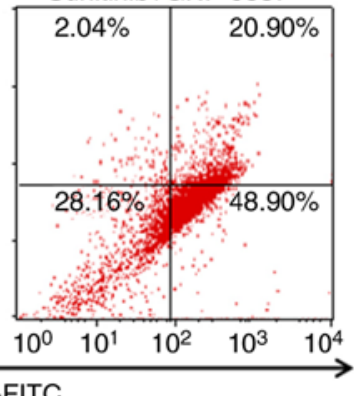

Annexin V-FITC

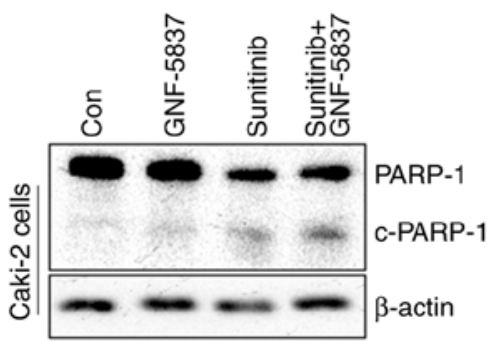

Figure 5. GNF-5837 cooperates with sunitinib to inhibit RCC. (A) 7860 and Caki-2 cells were incubated with increasing concentrations of GNF-5837 in the presence or absence of $20 \mu \mathrm{M}$ sunitinib for $24 \mathrm{~h}$. Cell viability was determined by MST assay. Data represent mean \pm SD of three independent experiments ${ }^{*} \mathrm{P}<0.05$, vs. the control (Con). (B) The proportion of apoptotic $786 \mathrm{O}$ cells was evaluated by flow cytometry using Annexin V-FITC/PI staining following treatment with $20 \mu \mathrm{M}$ sunitinib alone or in combination with $20 \mu \mathrm{M}$ GNF-5837 for $24 \mathrm{~h}$. (C) The influence of sunitinib and GNF-5837 (alone or both) on the cleavage of PARP-1 protein in 7860 and Caki-2 cells. RCC, renal cell carcinoma.

A

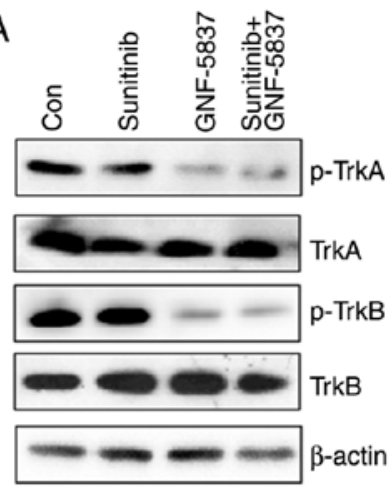

B

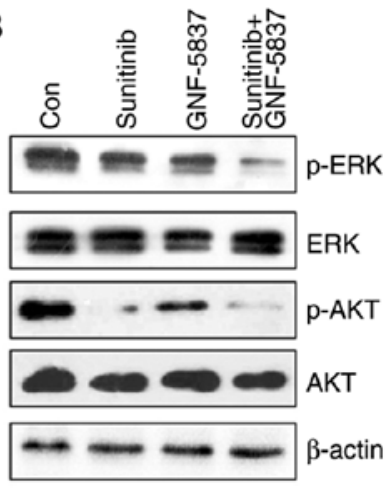

Figure 6. GNF-5837 cooperates with sunitinib to inhibit the ERK cascade. (A and B) $786 \mathrm{O}$ cells were incubated with $20 \mu \mathrm{M}$ GNF-5837 and/or $20 \mu \mathrm{M}$ sunitinib for $24 \mathrm{~h}$. Then western blot analysis was performed for the evaluation of phosphorylation levels (p-) of TrkA and TrkB (A) and ERK and AKT (B). Representative images from at least three independent experiments are shown.

alone did not affect the active levels of TrkA and TrkB kinases, which were greatly suppressed upon GNF-5837 treatment. In contrast, sunitinib effectively reduced the activities of ERK and AKT kinases (Fig. 6B). By comparison, the combined

treatment with sunitinib and GNF-5837 mainly yielded a cooperative inhibition on ERK activity. As the ERK cascade is critical for cell growth and survival, the synergistic action of GNF-5837 and sunitinib may exert cell toxicity via the repression of the ERK cascade.

GNF-5837 suppresses cell migration. About one-third of patients with RCC present with metastatic disease at diagnosis. Metastatic RCCs are poorly responsive to treatment and have a poorer prognosis. Pharmacological modulation of tumor metastasis contributes to improved clinical outcome. Indeed, Trk signaling combined with neurotrophins is reported to stimulate cell migration and invasion $(9,10)$. Thus, we performed wound healing assay to assess whether GNF-5837 inhibits cell migration. As shown in Fig. 7A and B, GNF-5837 treatment was able to reduce the migratory distance of 7860 and Caki-2 cells compared with the control group. As one small GTPase, Rac1, mediates cytoskeleton rearrangements to facilitate synaptic plasticity and tumor metastasis, we further investigated the effect of GNF-5837 on Racl activity. As shown in Fig. 7C, GNF-5837 treatment reduced the phosphorylation level of Rac1, accounting for the impaired cell migration. 
A
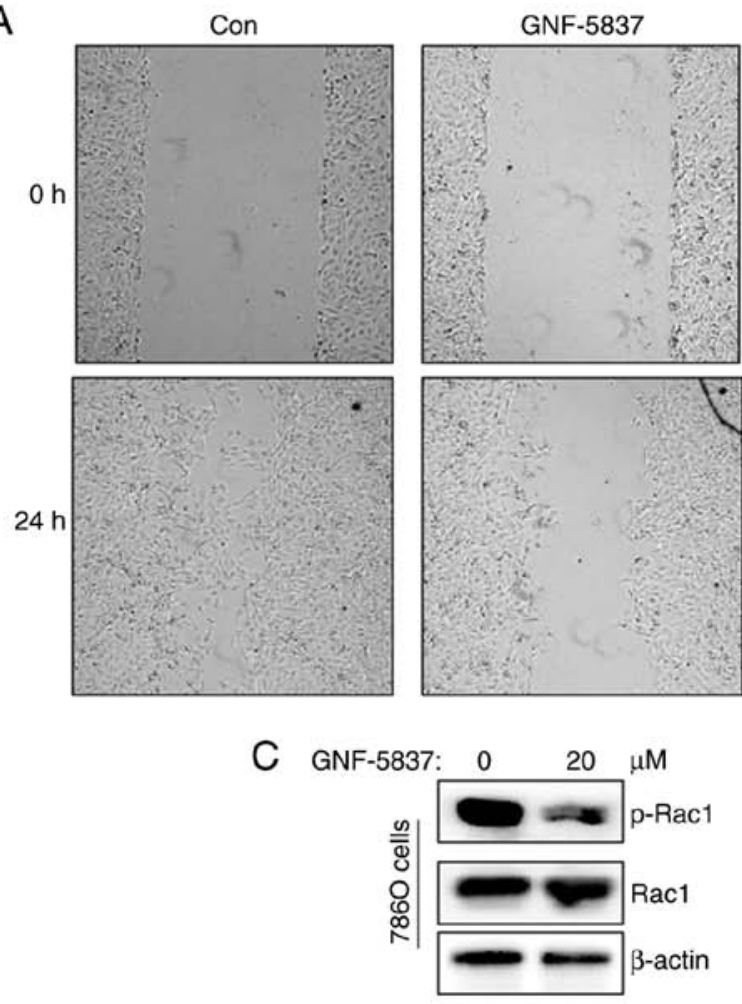

B
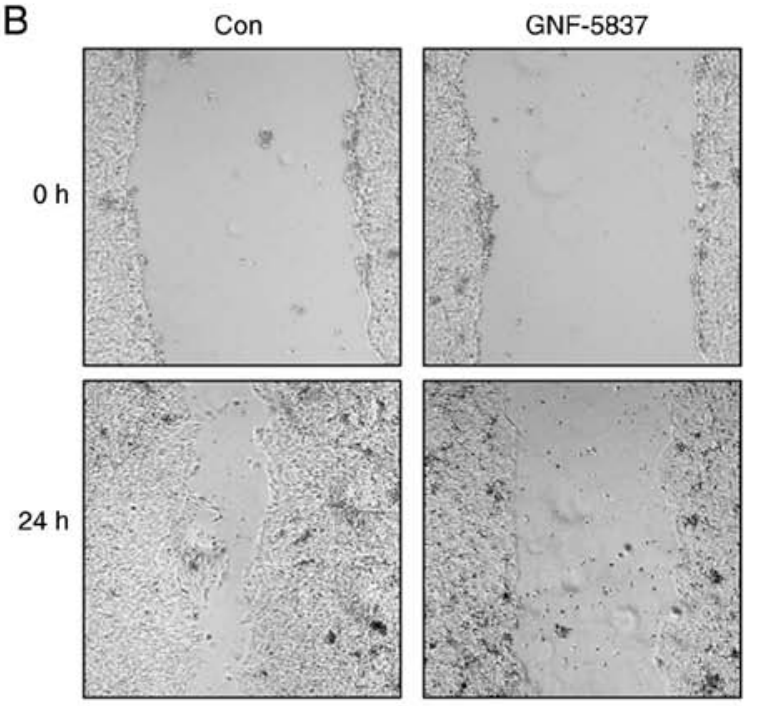

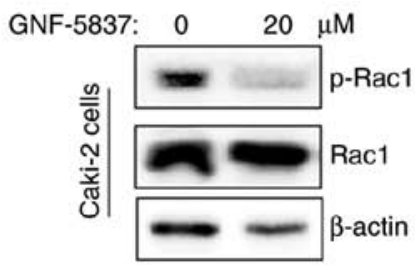

Figure 7. GNF-5837 inhibits cell migration. (A and B) Wound healing assays were performed on $786 \mathrm{O}$ and Caki-2 cells in the absence or the presence of $20 \mu \mathrm{M}$ GNF-5837. Then, representative images were captured at x10 magnification after treatment for $24 \mathrm{~h}$. (C) Western blot analysis was performed for the evaluation of the phosphorylation level (p-) of Racl after GNF-5837 treatment for $24 \mathrm{~h}$.

\section{Discussion}

RCC is a lethal and invasive tumor, and continues to present a major therapeutic challenge. Diverse growth factors and their corresponding kinase receptors are aberrantly expressed in this malignancy and contribute to tumor cell growth and invasion (11). Targeting growth factors and/or their receptors presents a promising therapeutic strategy for RCC. In the present study, we demonstrated that the pan-Trk inhibitor GNF-5837 exerts its cytotoxicity on RCC cells by inducing growth arrest and apoptosis.

Neurotrophins are growth factors that play important roles in neurogenesis during development and regeneration. The family of neurotrophins include nerve growth factor (NGF), brain derived neurotrophic factor (BDNF), neurotrophin-3 (NT-3) and neurotrophin-4/5 (NT-4/5) (12-14). Four mammalian neurotrophins bind to two distinct types of receptors: Tropomyosin receptor kinase (Trk) receptors and the p75 neurotrophin receptor (p75NTR) $(15,16)$. The Trk receptors are high-affinity NGF receptors and transmembrane tyrosine kinases. The Trk receptors include TrkA, TrkB and TrkC, each of which exhibits different specificities for neurotrophins $(17,18)$. NGF has preferential affinity for TrkA, BDNF and NT-4/5 for TrkB, and NT-3 for TrkC. The p75 neurotrophin receptor (p75NTR) is the low-affinity NGF receptor and belongs to the tumor necrosis factor (TNF) receptor superfamily (19).

Trk receptors activate and autophosphorylate tyrosine residues in their intracellular tails, triggering various downstream signaling pathways that involve cytoplasmic adaptors and enzymes such as extracellularly regulated kinases (ERKs), phosphatidyl inositol kinase 3 (PI3K) and phospholipase C (PLC) $(20,21)$. Binding of a neurotrophin to Trk receptors predominantly leads to promotion of cell survival, proliferation, and differentiation. Each neurotrophin also binds to p75NTR and activates multiple signaling pathways including the c-Jun N-terminal kinase (JNK) signaling cascade, which can enhance neuronal survival or induce cell death depending on cellular context $(15,22)$.

Neurotrophins also participate in physiological events outside of the nervous system, such as embryonic development, vascularization and organ homeostasis. In addition, increasing evidence has shown that neurotrophins and their receptors are involved in the growth, angiogenesis, dissemination, and drug resistance in different tumor types $(10,11)$. Neurotrophins and its receptors are abnormally overexpressed in lung, pancreatic, breast, liver, skin, ovarian, thyroid, and gastric tumors. For instance, primary and metastatic melanoma cell lines synthesize and secrete all neurotrophins and express both p75NTR and Trk receptors (11). Neurotrophin and their receptors are frequently found yielding an autocrine stimulation of tumor cell proliferation and metastasis $(12,19,20)$. It is well known that NGF/TrkA as a mitogenic signal promotes cell proliferation and invasion in breast cancer via signaling pathways similar to those implicated in neuronal development, such as ERK and AKT cascades (17). Additionally, NGF was found to modulate self-renewal and plasticity of tumor stem cells, and was found to be involved in the promotion of epithelial-mesenchymal transition (EMT) (22). In ovarian carcinomas, NGF activates TrkA in granulosa cells, where it functions as an indirect 
angiogenic factor by enhancing vascular endothelial growth factor (VEGF) expression, resulting in tumor cell proliferation, migration and vasculogenesis (18). BDNF is also known to participate in the cell division and dissemination of tumor cells through autocrine stimulation of TrkB and p75NTR. Aberrant BDNF/TrkB signaling is recognized as a driver and potential target in gastrointestinal cancer (13). Functional experiments have illuminated that BDNF administration promotes tumor cell proliferation, invasion and migration in both gastric and bowel cancer cell lines, as well as tumor cell metastasis in mouse models $(14,16)$.

Multiple studies $(11-13,15,18,20,22)$ have highlighted the potential utility of neurotrophins and their receptors as diagnostic and prognostic biomarkers in malignancies of diverse origins. Both TrkA and p75NTR are increased in thyroid cancer, and in particular TrkA expression is highly associated with lymph node invasion, implicating its involvement in tumor metastasis (15). NGF may serve as a biomarker of high-grade prostate cancer. Urinary NGF levels, as analyzed in a cohort of 115 prostate cancer patients, were found to be significantly correlated with tumor grade (Gleason score) and were thought to contribute to nerve infiltration in the tumor microenvironment (23). Studies in ovarian cancer have unveiled a dramatically increased expression of NGF, TrkA, and p75NTR in tumor tissues (21). In gastric cancer, BDNF is overexpressed and correlated with factors reflecting disease progression and poor prognosis, pointing to its value as a diagnostic and prognostic factor of clinical significance (13). Moreover, colorectal cancer patients with higher levels of BDNF and TrkB display greatly worse overall survival and elevated cancer metastasis (14).

However, the detailed effects of neurotrophin signaling in RCC largely remain unclear. Comprehensive molecular profiling has identified the abnormal activation of NGF signaling in metastatic clear cell renal cell carcinoma (24). A cohort study of 83 clear cell RCC tumors showed that overexpression of p75NTR, pro-BDNF, and to a lesser extent TrkB was detected by immunohistochemistry (16). In particular, p75NTR, mainly expressed in tumor tissues, was highly correlated with a higher Fuhrman grade in multivariate analysis. p75NTR inhibition by silencing or blocking antibody repressed cell survival and migration in RCC cell lines. Meanwhile, TrkB silencing caused apoptosis, inhibited proliferation, retarded invasion as well as improved antitumor efficiency of sorafenib in anoikis-resistant RCC cells (25).

Trk inhibitors such as KK5101, GNF-5837, AZD6918, GW441756 and GNF-4256, have exhibited suppressive effects on tumor proliferation, migration, and metastasis (26-30). In the present study, we observed that a pan-Trk inhibitor GNF-5837 exerted inhibitory effects on cell proliferation, survival and migration in RCC cells. GNF-5837 greatly suppressed the activation of TrkA and TrkB in 7860 and Caki-2 cells, thereby leading to the inhibition of downstream signals such as ERK and AKT kinases. As ERK and AKT kinases are important downstream modulators of Trk receptors, their inhibition should negatively regulate cell growth and survival. We further observed that GNF-5837 effectively induced G0/G1-phase arrest, increased p21 expression and downregulated c-Myc to achieve growth inhibition. qPCR analysis revealed that the expression of anti-apoptotic
Survivin was downregulated by GNF-5837. Conversely, the pro-apoptotic gene Bax was greatly induced following GNF-5837 treatment. Despite that, our results indicated that GNF-5837 is a moderate apoptosis inducer. Seemingly, GNF-5837 mainly exerts cell toxicity via inhibiting cell growth. In addition, neurotrophins are closely linked with cell migration of neural cells $(9,10)$. Herein, our results indicated that GNF-5837 also suppressed cell migration in RCC cells. Neurotrophin receptors have been shown to alter the expression of E-cadherin and Twist, thereby affecting epithelial-to-mesenchymal transition (EMT) and cell migration (31). BDNF-TrkB was sufficient to activate the Rho GTPase proteins Rac1 and $\mathrm{Cdc} 42$ to modulate cytoskeleton rearrangements, leading to the facilitation of synaptic plasticity (32). Our findings showed that GNF-5837 treatment impeded Rac1 activation, which contributed to the suppression of cell migration. Overall, our results further shed light on the putative effects of neurotrophin signaling in the pathological progress of RCC. Therefore, inhibition of neurotrophins and their receptors may be a novel potential therapeutic strategy for RCC. Despite that, it is essential to explore the origin of neurotrophins such as NGF and BDNF, and the precise role of distinct receptors in RCC. In addition, the present study was limited as it did not investigate the effects of GNF-5837 on normal renal cells.

In fact, a subset of preclinical trials have shown that blockade of neurotrophin/receptor by neutralizing antibody, siRNA-mediated silence, or pharmacological inhibition causes the inhibition of growth and invasion in a variety of human tumors. For instance, targeting NGF signaling via anti-NGF or anti-TrkA blocking antibodies has demonstrated tumor growth inhibition, reduced metastasis, and increased cancer cell apoptosis in breast cancer xenografted mice (33). Furthermore, siRNA-mediated knockdown of TrkA in breast cancer cells suppressed cancer cell growth and cell cycle progression with stagnation at G0/G1 phase, also increased chemo-sensitivity to paclitaxel and reduced the incidence of lung metastasis in xenograft models (34). Similarly, knockdown of TrkB in human lung adenocarcinoma cancer cell lines significantly decreased their migratory and metastatic ability in vitro and in vivo (35).

Tumor cells may escape the antagonism of VEGF signaling using other parallel pathways, such as neurotrophin signaling. Herein, we provided evidence that GNF-5837 can potentiate sunitinib-induced cytotoxicity via inhibition of TrkA and TrkB. Despite that, we lacked in vivo tumorigenic assessment and the combined effects on cell invasion. The combined treatment of GNF-5837 and sunitinib mainly interrupted ERK activity. Thus, the enhanced suppression on cell growth and survival may be attributed to ERK inhibition. In fact, a previous study noted that ERK signaling plays a role in the generation of sunitinib resistance in RCC patients receiving anti-angiogenic therapy (36). However, it is still unclear whether there is a synergistic effect of GNF-5837 and sunitinib. A series of experiments is needed to determine the combination index and associated mechanisms. Meanwhile, it is also obscure whether GNF-5837 can impede the generation of sunitinib resistance. Further investigation is still required to explore whether neurotrophins and their receptors are involved in sunitinib resistance. 


\section{Acknowledgements}

Not applicable.

\section{Funding}

The present study was supported by the Zhejiang Provincial Natural Science Foundation (grant no. LY18H160002).

\section{Availability of data and materials}

All data generated or analyzed during the present study are included in this published article.

\section{Authors' contributions}

GD and TL conceived and designed the study and the experiments. YiC acquired the funding. YiC, $\mathrm{HW}$ and $\mathrm{YuC}$ performed the experiments. MW analyzed the data and conducted the statistical analysis. TL wrote the manuscript. YiC and HW critically revised the manuscript. All authors read and approved the manuscript and agree to be accountable for all aspects of the research in ensuring that the accuracy or integrity of any part of the work are appropriately investigated and resolved.

\section{Ethics approval and consent to participate}

Not applicable.

\section{Patient consent for publication}

Not applicable.

\section{Competing interests}

These authors declare that they have no competing interests.

\section{References}

1. Hsieh JJ, Purdue MP, Signoretti S, Swanton C, Albiges L, Schmidinger M, Heng DY, Larkin J and Ficarra V: Renal cell carcinoma. Nat Rev Dis Primers 3: 17009, 2017.

2. Graves A, Hessamodini H, Wong G and Lim WH: Metastatic renal cell carcinoma: Update on epidemiology, genetics, and therapeutic modalities. Immunotargets Ther 2: 73-90, 2013.

3. Rodriguez-Vida A, Hutson TE, Bellmunt J and Strijbos MH: New treatment options for metastatic renal cell carcinoma. ESMO Open 2: e000185, 2017.

4. Mattei J, da Silva RD, Sehrt D, Molina WR and Kim FJ: Targeted therapy in metastatic renal carcinoma. Cancer Lett 343: 156-160, 2014.

5. da Silva RD, Gustafson D, Nogueira L, Werahera PN, Molina WR and Kim FJ: Targeted therapy for metastatic renal carcinoma: An update. J Kidney Cancer VHL 1: 63-73, 2014.

6. Posadas EM, Limvorasak S and Figlin RA: Targeted therapies for renal cell carcinoma. Nat Rev Nephrol 13: 496-511, 2017.

7. Chen $\mathrm{Y}$, Huang $\mathrm{Q}$, Zhou $\mathrm{H}$, Wang $\mathrm{Y}, \mathrm{Hu} \mathrm{X}$ and $\mathrm{Li} \mathrm{T}$ : Inhibition of canonical WNT/ $\beta$-catenin signaling is involved in leflunomide (LEF)-mediated cytotoxic effects on renal carcinoma cells. Oncotarget 7: 50401-50416, 2016.

8. Livak KJ and Schmittgen TD: Analysis of relative gene expression data using real-time quantitative PCR and the 2(-Delta Delta C(T) method. Methods 25: 402-408, 2001

9. Lee WD, Wang KC, Tsai YF, Chou PC, Tsai LK and Chien CL: Subarachnoid hemorrhage promotes proliferation, differentiation, and migration of neural stem cells via BDNF upregulation. PLoS One 11: e0165460, 2016.
10. Oliveira SL, Trujillo CA, Negraes PD and Ulrich H: Effects of ATP and NGF on proliferation and migration of neural precursor cells. Neurochem Res 40: 1849-1857, 2015.

11. Truzzi F, Marconi A, Lotti R, Dallaglio K, French LE, Hempstead BL and Pincelli C: Neurotrophins and their receptors stimulate melanoma cell proliferation and migration. J Invest Dermatol 128: 2031-2040, 2008.

12. Wang W, Chen J and Guo X: The role of nerve growth factor and its receptors in tumorigenesis and cancer pain. Biosci Trends 8: 68-74, 2014.

13. Okugawa Y, Tanaka K, Inoue Y,Kawamura M,Kawamoto A,Hiro J, Saigusa S, Toiyama Y, Ohi M,Uchida K, et al: Brain-derived neurotrophic factor/tropomyosin-related kinase B pathway in gastric cancer. Br J Cancer 108: 121-130, 2013

14. Tanaka K, Okugawa Y, Toiyama Y, Inoue Y, Saigusa S, Kawamura M, Araki T, Uchida K, Mohri Y and Kusunoki M: Brain-derived neurotrophic factor (BDNF)-induced tropomyosin-related kinase B (Trk B) signaling is a potential therapeutic target for peritoneal carcinomatosis arising from colorectal cancer. PLoS One 9: e96410, 2014.

15. Faulkner S, Jobling P, Rowe CW, Rodrigues Oliveira SM, Roselli S, Thorne RF, Oldmeadow C, Attia J, Jiang CC, Zhang XD, et al: Neurotrophin receptors TrkA, p75 $5^{\mathrm{NTR}}$, and sortilin are increased and targetable in thyroid cancer. Am J Pathol 188: 229-241, 2018.

16. De la Cruz-Morcillo MA, Berger J, Sánchez-Prieto R, Saada S, Naves T, Guillaudeau A, Perraud A, Sindou P, Lacroix A, Descazeaud A, et al: p75 neurotrophin receptor and pro-BDNF promote cell survival and migration in clear cell renal cell carcinoma. Oncotarget 7: 34480-34497, 2016.

17. Lagadec C, Meignan S, Adriaenssens E, Foveau B, Vanhecke E, Romon R, Toillon RA, Oxombre B, Hondermarck $\mathrm{H}$ and Le Bourhis X: TrkA overexpression enhances growth and metastasis of breast cancer cells. Oncogene 28: 1960-1970, 2009.

18. Vera C, Tapia V, Vega M and Romero C: Role of nerve growth factor and its TRKA receptor in normal ovarian and epithelial ovarian cancer angiogenesis. J Ovarian Res 7: 82, 2014.

19. Griffin N, Faulkner S, Jobling P and Hondermarck H: Targeting neurotrophin signaling in cancer: The renaissance. Pharmacol Res 135: 12-17, 2018.

20. Demir IE, Tieftrunk E, Schorn S, Friess H and Ceyhan GO: Nerve growth factor \& TrkA as novel therapeutic targets in cancer. Biochim Biophys Acta 1866: 37-50, 2016.

21. Yu X, Liu Z, Hou R, Nie Y and Chen R: Nerve growth factor and its receptors on onset and diagnosis of ovarian cancer. Oncol Lett 14: 2864-2868, 2017.

22. Tomellini E, Touil Y,Lagadec C, Julien S, Ostyn P,Ziental-Gelus N, Meignan S, Lengrand J, Adriaenssens E, Polakowska R and Le Bourhis X: Nerve growth factor and proNGF simultaneously promote symmetric self-renewal, quiescence, and epithelial to mesenchymal transition to enlarge the breast cancer stem cell compartment. Stem Cells 33: 342-353, 2015.

23. Liss MA, Gordon A, Morales B, Osann K, Skarecky D, Lusch A, Zaldivar F and Ahlering TE: Urinary nerve growth factor as an oncologic biomarker for prostate cancer aggressiveness. Urol Oncol 32: 714-719, 2014

24. Ghatalia P, Yang ES, Lasseigne BN, Ramaker RC, Cooper SJ, Chen D, Sudarshan S, Wei S, Guru AS, Zhao A, et al: Kinase gene expression profiling of metastatic clear cell renal cell carcinoma tissue identifies potential new therapeutic targets. PLoS One 11: e0160924, 2016.

25. Zhang P, Xing Z, Li X, Song Y, Zhao J, Xiao Y and Xing Y: Tyrosine receptor kinase $B$ silencing inhibits anoikis-resistance and improves anticancer efficiency of sorafenib in human renal cancer cells. Int J Oncol 48: 1417-1425, 2016.

26. Fang Z, Han B, Jung KH, Lee JH, El-Damasy AK, Gadhe CG, Kim SJ, Yan HH, Park JH, Lee JE, et al: A novel tropomyosin-related kinase A inhibitor, KK5101 to treat pancreatic cancer. Cancer Lett 426: 25-36, 2018.

27. Albaugh P, Fan Y, Mi Y, Sun F, Adrian F, Li N, Jia Y, Sarkisova Y, Kreusch A, Hood T, et al: Discovery of GNF-5837, a selective TRK inhibitor with efficacy in rodent cancer tumor models. ACS Med Chem Lett 3: 140-145, 2012.

28. Li Z, Zhang Y, Tong Y, Tong J and Thiele CJ: Trk inhibitor attenuates the BDNF/TrkB-induced protection of neuroblastoma cells from etoposide in vitro and in vivo. Cancer Biol Ther 16: 477-483, 2015.

29. Croucher JL, Iyer R, Li N, Molteni V, Loren J, Gordon WP, Tuntland T, Liu B and Brodeur GM: TrkB inhibition by GNF-4256 slows growth and enhances chemotherapeutic efficacy in neuroblastoma xenografts. Cancer Chemother Pharmacol 75: 131-141, 2015. 
30. Bapat AA, Munoz RM, Von Hoff DD and Han H: Blocking nerve growth factor signaling reduces the neural invasion potential of pancreatic cancer cells. PLoS One 11: e0165586, 2016.

31. Kupferman ME, Jiffar T, El-Naggar A, Yilmaz T, Zhou G, Xie T, Feng L, Wang J, Holsinger FC, Yu D and Myers JN: TrkB induces EMT and has a key role in invasion of head and neck squamous cell carcinoma. Oncogene 29: 2047-2059, 2010.

32. Hedrick NG, Harward SC, Hall CE, Murakoshi H, McNamara JO and Yasuda R: Rho GTPase complementation underlies BDNF-dependent homo- and heterosynaptic plasticity. Nature 538: 104-108, 2016.

33. Adriaenssens E, Vanhecke E, Saule P, Mougel A, Page A Romon R, Nurcombe V, Le Bourhis X and Hondermarck H: Nerve growth factor is a potential therapeutic target in breast cancer. Cancer Res 68: 346-351, 2008.
34. Zhang J, Wang LS, Ye SL, Luo P and Wang BL: Blockage of tropomyosin receptor kinase a (TrkA) enhances chemo-sensitivity in breast cancer cells and inhibits metastasis in vivo. Int J Clin Exp Med 8: 634-641, 2015.

35. Sinkevicius KW, Kriegel C, Bellaria KJ, Lee J, Lau AN, Leeman KT, Zhou P, Beede AM, Fillmore CM, Caswell D, et al: Neurotrophin receptor TrkB promotes lung adenocarcinoma metastasis. Proc Natl Acad Sci USA 111: 10299-10304, 2014

36. Diaz-Montero CM, Mao FJ, Barnard J, Parker Y, Zamanian-Daryoush M, Pink JJ, Finke JH, Rini BI and Lindner DJ: MEK inhibition abrogates sunitinib resistance in a renal cell carcinoma patient-derived xenograft model. $\mathrm{Br}$ J Cancer 115: 920-928, 2016. 